\title{
Momenta of a vortex tangle by structural complexity analysis
}

\author{
Renzo L. Ricca* \\ Department of Mathematics and Applications, University of Milano-Bicocca, Via Cozzi 53, 20125 Milano, Italy \\ Institute for Scientific Interchange, Villa Gualino, 10133 Torino, Italy
}

Available online 6 January 2008

\begin{abstract}
A geometric method based on information from structural complexity is presented to calculate linear and angular momenta of a tangle of vortex filaments in Euler flows. For thin filaments under the so-called localized induction approximation the components of linear momentum admit interpretation in terms of projected area. By computing the signed areas of the projected graph diagrams associated with the vortex tangle, we show how to calculate the two momenta of the system by complexity analysis of tangle diagrams. This method represents a novel technique to extract dynamical information of complex systems from geometric and topological properties and provides a potentially useful tool to test the accuracy of numerical methods and investigate scale distribution of fluid dynamical properties of vortex flows.
\end{abstract}

(C) 2008 Elsevier B.V. All rights reserved.

PACS: 47.10.A-; 47.10.ab; 47.32.C-; 47.37.+q

Keywords: Vortex tangle; Structural complexity

\section{Conservation of linear and angular momenta of a vortex tangle}

In this paper we present new mathematical results concerning a method based on signed area of oriented graphs developed to evaluate linear and angular momenta of a tangle of vortex filaments in Euler's flows. In paying our tribute to celebrate more than 250 years of work on Euler's equations, we are particularly happy to present and discuss here new ideas that rely not only on such a fruitful setting, but also on another Euler's remarkable contribution, rooted in his 1735 solution of the famous Königsberg's Bridge Problem [1], namely the foundation of graph theory and what, arguably, we now call topology [2]. The idea of using graph theoretical information to study fluid dynamical properties was originally put forward by Kelvin in 1867 [3], but it remained little explored. What we present here benefits from the progress made in recent years in algebraic topology and geometric fluid mechanics and,

\footnotetext{
* Corresponding address: Department of Mathematics and Applications, University of Milano-Bicocca, Via Cozzi 53, 20125 Milano, Italy. Fax: +39 0264485705 .

E-mail address: renzo.ricca@unimib.it.
}

we believe, has great potential for further developments and future applications in visiometric diagnostics of structural flow complexity [4,5].

For simplicity, let us consider the evolution of a vortex tangle in an unbounded, ideal fluid at rest at infinity, where vorticity remains localized on thin filaments of infinitesimal cross-sections. Such vortex tangles arise naturally in superfluid turbulence [6], where indeed vorticity remains confined on very thin filaments for very long time. In this context vortex evolution may be approximated by the so-called localized induction approximation, LIA for short [7,8]. The analytical results presented here are rigorously valid for LIA evolutions, and can, under mild assumptions, be adapted to evolutions of vortex filaments governed by the full Biot-Savart law (see the last section for a brief clarifying comment).

It is well-known that LIA is directly related to the nonlinear Schrödinger equation, that in one dimension is completely integrable, preserving an infinity of invariants of motion. It is remarkable that among such invariants two classical invariants of the Euler equations survive, namely the linear and angular momenta $[9,10]$. Let us consider these invariants for a vortex tangle. Let $\mathcal{T}=\left\{\bigcup_{i} \mathcal{L}_{i}\right\}_{i=1, \ldots, N}$ denote a tangle of $N$ vortex lines $\mathcal{L}_{i}$, each line being a smooth curve in $\mathbb{R}^{3}$, parametrized by 

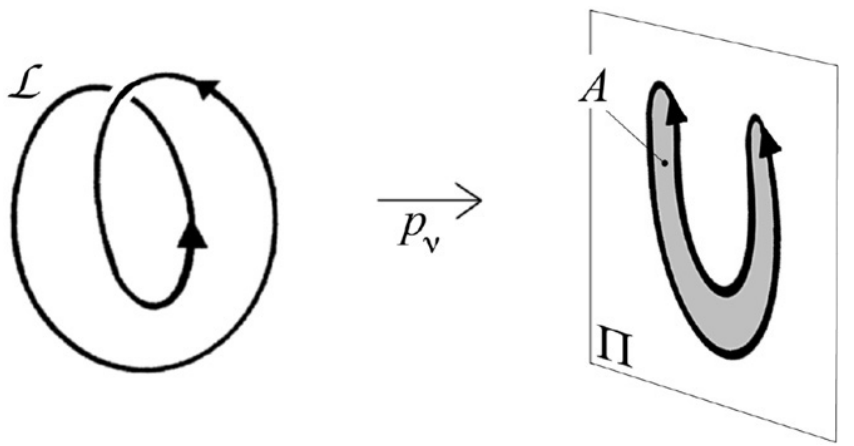

Fig. 1. The area $A$ of the projected graph resulting from the projection $p_{v}$ of a vortex line $\mathcal{L}$ on the plane $\Pi$ is proportional to the component of the linear momentum of $\mathcal{L}$ in the $\boldsymbol{v}$-direction.

arc-length $s$. Vorticity $\boldsymbol{\omega}$ is defined on $\mathcal{L}_{i}$, and is simply given by $\boldsymbol{\omega}=\bar{\omega} \mathbf{X}^{\prime}$, where, in general, $\mathbf{X}=\mathbf{X}(s, t)$ denotes the position vector, $\bar{\omega}$ a constant and $\mathbf{t} \equiv \mathbf{X}^{\prime}$ the unit tangent to $\mathcal{L}_{i}$ (the prime denoting the derivative with respect to $s$, and $t$ is time). The linear momentum $\mathbf{P}=\mathbf{P}(\mathcal{T})$ corresponds to the hydrodynamic impulse, which generates the motion of $\mathcal{T}$ from rest, and from its standard definition [11] takes the form

$\mathbf{P}=\frac{1}{2} \int_{\mathcal{T}} \mathbf{X} \times \omega^{3} \mathrm{~d}^{3} \mathbf{X}=\frac{1}{2} \sum_{i=1}^{N} \Gamma_{i} \int_{\mathcal{L}_{i}} \mathbf{X} \times \mathbf{X}^{\prime} \mathrm{d} s$,

where $\mathbf{P}$ is here intended per unit density, and $\Gamma_{i}$ represents the circulation of $\mathcal{L}_{i}$. Similarly, for the angular momentum $\mathbf{M}=\mathbf{M}(\mathcal{T})$, that corresponds to the moment of the impulsive forces acting on $\mathcal{T}$; we have

$$
\begin{aligned}
\mathbf{M} & =\frac{1}{3} \int_{\mathcal{T}} \mathbf{X} \times(\mathbf{X} \times \boldsymbol{\omega}) \mathrm{d}^{3} \mathbf{X} \\
& =\frac{1}{3} \sum_{i=1}^{N} \Gamma_{i} \int_{\mathcal{L}_{i}} \mathbf{X} \times\left(\mathbf{X} \times \mathbf{X}^{\prime}\right) \mathrm{d} s,
\end{aligned}
$$

where, again, $\mathbf{M}$ is intended per unit density. We remark that under both Euler's equations and LIA, we have

$$
\frac{\mathrm{d} \mathbf{P}}{\mathrm{d} t}=0, \quad \frac{\mathrm{d} \mathbf{M}}{\mathrm{d} t}=0 .
$$

\section{Interpretation of momenta in terms of projected area}

Arms and Hama [8], who first proved the conservation of the integral on the right-hand side of Eq. (1) for a single vortex line, showed that this quantity admits interpretation in terms of projected area (see Fig. 1). Indeed, by direct inspection of the integrand above, it is evident that under LIA the plane projected area of the vortex line is proportional to the component of the linear momentum of the vortex along the direction of projection.

Let $p=p_{v}$ denote the orthogonal projection onto the plane $\Pi$ along the direction $\boldsymbol{v}$, and $\mathcal{L}_{v}=p_{v}(\mathcal{L})$ be the graph diagram of a smooth space curve $\mathcal{L}$ under $p_{v}$. Evidently $\mathcal{L}_{v}$ depends on v. For the moment let $\mathcal{L}_{v}$ be a smooth planar curve with no self-intersections, but in general $\mathcal{L}_{v}$ will be a nodal curve with self-intersections, the latter resulting from the projection of the apparent crossings of $\mathcal{L}$, when $\mathcal{L}$ is viewed along the line of sight $v$.

By identifying the vortex line with its geometric support $\mathcal{L}$, the projected graph diagram $\mathcal{L}_{v}$ will be oriented, the orientation being naturally induced by the vorticity vector. Let $\mathcal{L}_{x y}, \mathcal{L}_{y z}$, $\mathcal{L}_{z x}$ be the three graph diagrams of the projected vortex line onto the mutually orthogonal planes $x=0, y=0, z=0$, and let $A_{x y}=A\left(\mathcal{L}_{x y}\right), A_{y z}=A\left(\mathcal{L}_{y z}\right), A_{z x}=A\left(\mathcal{L}_{z x}\right)$ be the corresponding areas of the plane regions bounded by $\mathcal{L}_{x y}, \mathcal{L}_{y z}$, $\mathcal{L}_{z x}$, respectively. have

By applying the results of Arms and Hama [8], from (1) we

$P_{x y}=\Gamma A_{x y}, \quad P_{y z}=\Gamma A_{y z}, \quad P_{z x}=\Gamma A_{z x}$,

where $\Gamma$ is vortex circulation. Moreover:

Definition 2.1. The resultant area $A_{\max }$ is the maximal area obtained by $\max _{v} p(A)=p_{\max }(A)$ (along the resultant axis $\boldsymbol{v}_{\max }$ ) among all possible projected areas $A$.

The direction of the resultant axis $\boldsymbol{v}_{\max }$ is clearly that of the linear momentum. Hence, also from [8], we have

Theorem 2.2 (Maximal Area Interpretation). The resultant linear momentum of a vortex line $\mathcal{L}$, of circulation $\Gamma$, under LIA is given by $\mathbf{P}=\Gamma A_{\max } \boldsymbol{v}_{\max }$, where $A_{\max }$ is the resultant area. The projected area of $\mathcal{L}$ on any plane perpendicular to that of the resultant area is zero.

Similar results hold true for the angular momentum. With reference to the right-hand side of Eq. (2), the second integral can be interpreted in terms of areal moment, according to the following definition:

Definition 2.3. The areal moment around any axis is the product of the area $A$ multiplied by the distance $d$ between that axis and the axis $\mathbf{a}_{G}$, normal to $A$ through the centroid $G$ of $A$.

For a vortex line $\mathcal{L}$, the centroid $G$ of the projected area $A$ is the center weighted with respect to the vorticity distribution of $\mathcal{L}$. As for the linear momentum, the components of the angular momentum are determined by the areal moments:

$M_{x y}=\Gamma d_{z} A_{x y}, \quad M_{y z}=\Gamma d_{x} A_{y z}, \quad M_{z x}=\Gamma d_{y} A_{z x}$,

where evidently $d_{x}, d_{y}, d_{z}$ are the distances between the rotational axis and the centroid axes through $A_{y z}, A_{z x}, A_{x y}$, respectively. Similar considerations apply to define the resultant areal moment of $\mathcal{L}$ :

Definition 2.4. The resultant areal moment of $\mathcal{L}$ is the areal moment around the resultant axis $\mathbf{a}_{G}$ of the projected areas of $\mathcal{L}$ onto two mutually orthogonal planes, parallel to $\mathbf{a}_{G}$.

These observations are easily extended to a tangle $\mathcal{T}=$ $\bigcup_{i} \mathcal{L}_{i}$ of $N$ vortex lines $\mathcal{L}_{i}$, provided we carefully define the 


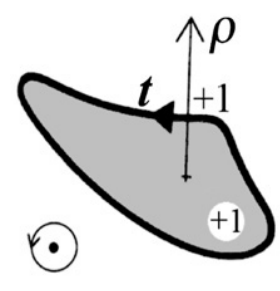

(a)

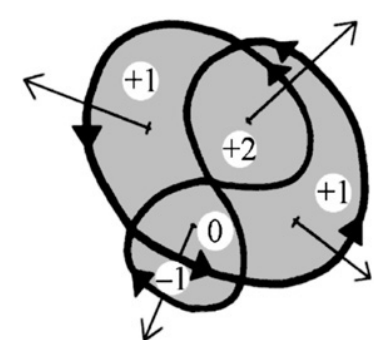

(b)

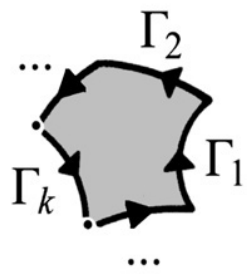

(c)

Fig. 2. (a) The number in the dashed region is the value of the index $\mathcal{I}_{P}(\mathcal{C})$ according to the right-hand rule convention and the algebraic intersection number calculated by Eq. (6). (b) The oriented nodal curve, resulting, for example, from the standard projection of a figure- 8 knot, has 5 bounded regions. Note that one of the interior regions has index 0 , due to the opposite orientation of the strands crossed by $\rho$. (c) Contribution from each $A\left(\mathcal{R}_{j}\right)$ must be weighted according to the circulations on the boundary $\partial \mathcal{R}_{j}$.

area of the resulting oriented graph diagram. The difficulty here is precisely in the correct calculation of such area.

\section{Signed area of oriented graph diagrams}

The oriented graph diagram of a tangle of vortex lines is an oriented nodal curve (i.e. the "underlying universe" of the tangle) in $\mathbb{R}^{2}$, and this can easily attain considerable complexity, particularly as regards the localization of selfintersections. A necessary first step is to reduce nodal curves of any complexity to good nodal curves, that have (at most) double points. Nodal points are classified according to their degree of multiplicity $\mu(P)$ given by the number of arcs incident at the point of intersection $P$. If $P$ is a double point, then $\mu(P)=2$. If $P$ is a point of multiplicity $\mu(P)=n(n>2)$, we can always reduce its multiplicity by "shaking" the graph diagram (actually its pre-image) near $P$ to get $m=\frac{1}{2}\left(n^{2}-n\right)$ double points, by virtual perturbations of the incident arcs from their location. Thus, if $h^{(n)}$ is the total number of points of multiplicity $n$, by applying this shaking technique we can always replace these $h^{(n)}$ points with $h(n)=m h^{(n)}(m \geq 3)$ double points. We say that a graph diagram is a good projection, when it has at most double points. Hence, by the shaking technique, we can always reduce highly complex graph diagrams to good nodal curves.

Let $\mathcal{C}$ denote one such good nodal curve on $\Pi$, and let $A(\mathcal{C})$ be the corresponding total area. In order to calculate this area, first we need to define the index $\mathcal{I}_{P}(\mathcal{C})$ of $\mathcal{C}$ at the point $P$ (for this see, for example, [12]). Let $P \notin \mathcal{C}, \mathbf{t}$ the tangent to $\mathcal{C}$ and $\rho$ the radiant vector with foot at $P$, that intersects $\mathcal{C}$ transversally. At each intersection point $X \in \rho \cap \mathcal{C}$ assign the algebraic sign $\epsilon(X)= \pm 1$, according to the standard convention given by the right-hand rule, that is $\epsilon(X)=+1$ when the frame $\{\boldsymbol{\rho}, \mathbf{t}\}$ is positive (see Fig. 2(a)). If $X$ is a double point, then the intersection is computed with one of the neighbouring pairs of the incident, equi-oriented arcs.

Definition 3.1. The index $\mathcal{I}_{P}(\mathcal{C})$ of $\mathcal{C}$ at $P$ is the algebraic intersection number given by

$\mathcal{I}_{P}(\mathcal{C})=\sum_{X \in \rho \cap \mathcal{C}} \epsilon(X)$.

Hence, $\mathcal{I}_{P}(\mathcal{C}) \in \mathbb{Z}$.
Let us now consider the $Z$ sub-domains $\left\{\mathcal{R}_{j}\right\}_{j=1, \ldots, Z}$ determined by $\mathcal{C} \cap \Pi$ and bounded by $\mathcal{C}$, and let $A\left(\mathcal{R}_{j}\right)>0$ denote their standard area. Since every point $P \in \mathcal{R}_{j}$ has the same $\mathcal{I}_{P}(\mathcal{C})$, we shall call $\mathcal{I}_{j}$ the index associated with any point $P \in \mathcal{R}_{j}$ and assign this value to each sub-domain $\mathcal{R}_{j}$ of $\mathcal{C} \cap \Pi$ (see Fig. 2(b)). The signed area of an oriented graph, a concept that can be traced back to Gauss [13], is thus given by the following rule.

Rule 3.2 (Signed Area). The signed area $A(\mathcal{C})$ of an oriented, planar nodal curve $\mathcal{C}$, is given by

$A(\mathcal{C})=\sum_{j=1}^{Z} \mathcal{I}_{j} A\left(\mathcal{R}_{j}\right)$,

where $A\left(\mathcal{R}_{j}\right)>0$ is the standard area of $\mathcal{R}_{j}$.

\section{Linear and angular momenta of a vortex tangle by structural complexity analysis}

By the signed area rule we can calculate the projected area of any nodal curve, be it the graph of a single vortex line, or that of a complex tangle of vortices. If the vortices have different circulations, a weighting factor defined in terms of contributions from each arc of $\partial \mathcal{R}_{j}$ must be assigned to $A\left(\mathcal{R}_{j}\right)$ (see Fig. 2(c)). The simplest correction comes from the algebraic weighting $\gamma_{j}$. Let $L_{j}=L\left(\partial \mathcal{R}_{j}\right)=\sum_{k=1, \ldots, M} L_{k, j}$ denote the total length of the boundary curve $\partial \mathcal{R}_{j}$ made of $M$ oriented arcs, the $k$-th arc having length $L_{k, j}$ and circulation $\Gamma_{k}$. We have

Definition 4.1. The circulation weighting factor $\gamma_{j}$ of $\mathcal{R}_{j}$ is given by

$\gamma_{j}=\frac{\sum_{k=1}^{M} \Gamma_{k} L_{k, j}}{L_{j}}$.

If all the vortices have same circulation $\Gamma$, then evidently $\gamma_{j}=\Gamma$. Appropriate weighting of circulation is necessary to determine the correct location of the centroid of the projected area. To summarize, we have the following result. 
Theorem 4.2 (Signed Area Interpretation). Let $\mathcal{T}$ be a vortex tangle evolving under LIA. Then, the linear momentum $\mathbf{P}=\mathbf{P}(\mathcal{T})$ has components

$P_{x y}=\sum_{j=1}^{Z} \gamma_{j} \mathcal{I}_{j} A_{x y}\left(\mathcal{R}_{j}\right), \quad P_{y z}=\cdots, \quad P_{z x}=\cdots$,

and the angular momentum $\mathbf{M}=\mathbf{M}(\mathcal{T})$ has components

$M_{x y}=d_{z} \sum_{j=1}^{Z} \gamma_{j} \mathcal{I}_{j} A_{x y}\left(\mathcal{R}_{j}\right), \quad M_{y z}=\cdots, \quad M_{z x}=\cdots$,

where $A_{x y}\left(\mathcal{R}_{j}\right), \ldots$, etc. denotes standard area of $\mathcal{R}_{j}$.

Proof of the above theorem is based on direct applications of (4) and (5), by using the signed area Rule 3.2.

\section{Dynamical aspects based on signed area information}

Signed area contributions provide useful information, that can be applied, predictively, to estimate and, postdictively, to understand some dynamical properties of the system. Remember that: (i) areas with index 0 do not contribute to the momentum; (ii) areas with high index (in absolute value) weight more and, proportionally, contribute more to the dynamical impulse of the system; (iii) areas of opposite sign determine contributions in opposite directions. Additional information on dynamical aspects may also come from index gradient analysis. Take the case of Fig. 2(b): here the alignment of regions, where the index gradually changes from -1 to +2 , indicates the presence of a principal axis of revolution. The exact location of this axis, placed orthogonally to the alignment of such regions, is determined by an accurate estimate of the weighted areas and, in any case, it can be determined by signed area information.

Let us consider two other examples, assuming, for simplicity, equal circulations and maximal projected areas. In Fig. 3(a) we have the projection of a single coiled filament, that in space is wound up 5 times around a circular axis (not shown). Contribution from the 5 negative areas exceeds that from the positive area, hence the resultant momentum is oriented in the negative direction. If such a vortex configuration existed, it would propagate backwardly in space. Such an unusual behaviour may not be so unrealistic, as recent analytical solutions $[14,15]$ and numerical tests [16] seem to suggest.

Another interesting case is illustrated by the following example. Consider the head-on collision of two anti-parallel vortex rings, propagating co-axially one against the other. The linear momentum of the two-ring system (as a whole) is obviously zero, and in ideal conditions this value is conserved until collisional time. In the case of real dynamics at sufficiently high Reynolds number, slight perturbations of the circular axes are likely to develop and, upon collision, we can expect that these will trigger sinusoidal disturbances along the two colliding ring axes. Here, analysis of the projected diagram may be rather illuminating. Without loss of generality and for the sake of simplicity, let us drastically simplify the situation and consider the perturbed circular axes as the elliptical curves
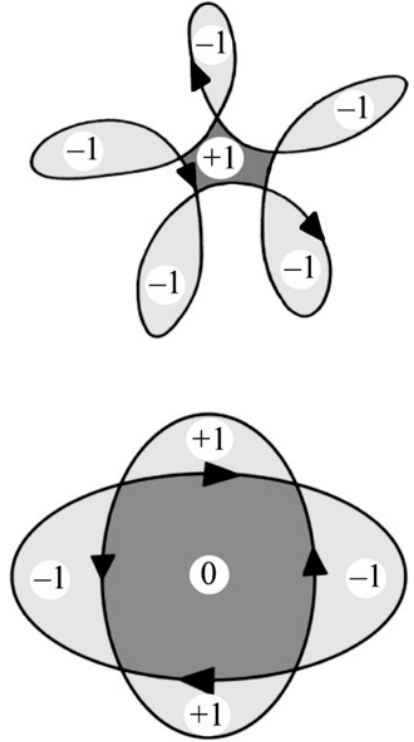

Fig. 3. (a) Projected diagram of a coiled vortex filament: contribution from negative areas (light grey) exceeds that from positive area (dark grey); hence, the resultant linear momentum of the vortex is negative. (b) Graph of the projection of two anti-parallel, elliptical vortex rings: opposite contributions from regions of index of alternating sign (light grey) cancel out; hence, the resultant linear momentum of the two-ring system is zero.

sketched in Fig. 3(b) (realistic perturbations would obviously generate a far more complex diagram). The central region has index 0 and is surrounded by four regions (light grey) of alternating sign. Consistently with what we expect from the annihilation of the rings velocity, this gives zero contribution to the resultant linear momentum of the two-ring system.

When the two rings collide, the alternating sign of the four surrounding regions is an indicator of an imminent structural instability, that produces the shoot-off of a pair of small vortex rings on either side of the collisional plane. In a realistic scenario, the precise number of surrounding regions will depend on geometric details of the perturbation, but in any case the production of an equal number of secondary small rings on either side of the collisional plane must be expected. These considerations seem confirmed by direct inspection of experimental results (see, for instance, [17]). In real experiments a diadem of a large number of secondary small vortex rings is clearly visible. This diadem grows from the instability of a fluid membrane that is produced in the collisional plane, upon collision of the primary large vortex rings. These secondary rings appear to be alternately distributed on either side of the collisional plane, surviving just for a short time before final dissipation.

\section{Concluding remarks}

The geometric method based on the signed area interpretation summarized by Theorem 4.2 provides a new and potentially useful tool for fluid dynamics research. The method exploits information from structural complexity analysis of a tangle of vortex filaments to estimate linear and angular momenta of the system. This is done by computing the signed areas of the projected graph diagrams associated with the vortex tangle, 
after application of appropriate shaking and weighting techniques. The results are rigorously valid in the LIA context, but, as mentioned earlier, in principle they could be extended to thin vortex filaments governed by the Biot-Savart law. This extension seems plausible as long as vorticity remains localized in a tubular domain of volume small compared with the fluid volume 'embraced' by the tangle. In terms of projected areas, this corresponds to assuming that the (standard) area of the vorticity domain is much smaller compared with the overall area enclosed by the outermost boundary projected curve, the order of approximation depending presumably on this ratio. Physically, this simply means that the higher the localization of vorticity, the most efficacious is the production and transfer of hydrodynamic impetus and moment, two quantities that are conserved under Euler's equations, regardless of the validity of the localized induction approximation.

In any case, for LIA systems the geometric method proposed here provides a potentially useful tool for predictive and postdictive diagnostics. By analyzing projected areas, it can be applied to implement tests of accuracy of numerical methods simulating vortex tangles. In superfluids, in particular, by analyzing the area distribution of the vortex projection one can judge about the scale distribution of linear and angular momenta, and compare this with the expected values of the spectrum of turbulence (Kolmogorov's twothirds law). Moreover, since LIA preserves an infinity of invariants of motion, all of these admitting a geometric interpretation in terms of global curvature, torsion and higherorder gradients $[18,10]$, these can be implemented to supply further information on dynamical properties (for instance, kinetic energy and helicity). Other features associated with the analysis of projected graphs can be related to dynamical issues, but this is beyond the scope of this article. We just like to conclude mentioning that the famous relation [19] $\chi(G)=$ $v-e+r$, between the Euler characteristic $\chi(G)$ of a graph $G$ (associated with vortex topology), of $v$ vertices, $e$ edges and $r$ regions, may find also useful applications in the study of complex systems [20] and in the advanced diagnostics of complex flow patterns.

\section{Acknowledgments}

I would like to thank C. Weber for pointing out Ref. [13]. Financial support from Italy's MIUR (D.M. 26.01.01, n. 13
"Incentivazione alla mobilità di studiosi stranieri e italiani residenti all'estero") and from ISI-Fondazione CRT (Lagrange Project) is kindly acknowledged.

\section{References}

[1] L. Euler, Solutio problematis ad geometriam situs pertinentis, Commentarii Academie Scientiarum Imperialis Petropolitanae 8 (1741) 128-140.

[2] G.L. Alexanderson, Euler and Königsberg's bridges: A historical view, Bull. Am. Math. Soc. 43 (2006) 567-573.

[3] Lord Kelvin (W. Thomson), On vortex atoms, Proc. R. Soc. Edin. 6 (1867) 94-105.

[4] C.F. Barenghi, R.L. Ricca, D.C. Samuels, How tangled is a tangle? Physica D 157 (2001) 197-206.

[5] R.L. Ricca, Structural complexity, in: A. Scott (Ed.), Encyclopedia of Nonlinear Science, New York and London, Routledge, 2005, pp. 885-887.

[6] K.W. Schwarz, Three-dimensional vortex dynamics in superfluid ${ }^{4} \mathrm{He}$ : Homogeneous superfluid turbulence, Phys. Rev. B 38 (1988) 2398-2417.

[7] L.S. Da Rios, On the motion of an unbounded liquid with a vortex filament of any shape, Rend. Circ. Mat. Palermo 22 (1906) 117-135 (in Italian).

[8] R.J. Arms, F.R. Hama, Localized induction concept on a curved vortex and motion of an elliptic vortex ring, Phys. Fluids 8 (1965) 553-559.

[9] Y. Fukumoto, On integral invariants for vortex motion under the localized induction approximation, J. Phys. Soc. Japan 56 (1987) 4207-4209.

[10] R.L. Ricca, Physical interpretation of certain invariants for vortex filament motion under LIA, Phys. Fluids A 4 (1992) 938-944.

[11] P.G. Saffman, Vortex Dynamics, Cambridge University Press, Cambridge, 1991.

[12] R. Langevin, Differential geometry of curves and surfaces, in: R.L. Ricca (Ed.), An Introduction to the Geometry and Topology of Fluid Flows, in: NATO Science Series II, vol. 47, Kluwer Academic Publs., Dordrecht, 2001, pp. 13-33.

[13] C.F. Gauss, Letter to H. Olbers, Werke 8 (1825) 398-400.

[14] Y. Fukumoto, Stationary configurations of a vortex filament in background flows, Proc. R. Soc. Lond. A 453 (1997) 1205-1232.

[15] L. Kiknadze, Yu. Mamaladze, The waves on the vortex ring in He II, J. Low Temp. Phys. 126 (2002) 321-326.

[16] C.F. Barenghi, R. Hänninen, M. Tsubota, Anomalous translational velocity of vortex ring with finite-amplitude Kelvin waves, Phys. Rev. E 74 (2006) 1-5.

[17] T.T. Lim, T.B. Nickels, Instability and reconnection in head-on collision of two vortex rings, Nature 357 (1992) 225-227.

[18] H.K. Moffatt, R.L. Ricca, Interpretation of invariants of the Betchov-Da Rios equations and of the Euler equations, in: J. Jimenez (Ed.), The Global Geometry of Turbulence, Plenum Press, New York, 1992, pp. 257-264.

[19] W.S. Massey, Algebraic Topology: An Introduction, Harcourt, Brace and World, Inc., New York.

[20] R.L. Ricca, Structural complexity and dynamical systems, in: R.L. Ricca (Ed.), Lectures on Topological Fluid Mechanics, Springer-CIME Lecture Notes in Mathematics, Springer-Verlag, Berlin, 2008 (in press). 